\title{
Efeito da condimentação permanente com alho desidratado e in natura nas características físico-químicas, atividade antioxidante e estabilidade térmica do azeite
}

\section{de oliva}

\author{
Effect of permanent aromatization with dehydrated and in natura garlic on the physicochemical \\ characteristics, antioxidant activity and thermal stability of olive oil \\ Efecto de la aromatización permanente con ajo deshidratado e in natura sobre las características \\ fisicoquímicas, actividad antioxidante y estabilidad termica del aceite de oliva
}

Recebido: 07/11/2021 | Revisado: 15/11/2021 | Aceito: 19/11/2021 | Publicado: 29/11/2021

\author{
Clédina de Oliveira Stiegemaier dos Santos \\ ORCID: https://orcid.org/0000-0001-7506-7902 \\ Universidade do Estado de Santa Catarina, Brasil \\ E-mail: cledina.de.oliveira@gmail.com \\ Taline Laura Bortolossi \\ ORCID: https://orcid.org/0000-0003-4572-4435 \\ Universidade do Estado de Santa Catarina, Brasil \\ E-mail: taline.bortolossi@edu.udesc.br \\ Andréia Zilio Dinon \\ ORCID: https://orcid.org/0000-0002-0676-5050 \\ Universidade do Estado de Santa Catarina, Brasil \\ E-mail: andreia.dinon@udesc.br
}

\begin{abstract}
Resumo
O alho (Allium sativum) é popularmente utilizado para condimentar azeites de oliva, possui altos teores de alicina e selênio com capacidade antioxidante, além de compostos fenólicos como os ácidos p-cumárico e cafeico. O presente trabalho avaliou a qualidade do azeite de oliva extravirgem condimentado individualmente com alho desidratado e com alho in natura, ambos na proporção de $10 \%(\mathrm{~m} / \mathrm{m})$ durante a infusão permanente por 50 dias à $60 \pm 2^{\circ} \mathrm{C}$. Foram realizadas análises para determinar a atividade antioxidante, o conteúdo de compostos fenólicos totais, a estabilidade térmica e os principais parâmetros de qualidade pela determinação do índice de acidez, índice de peróxidos, coeficiente de extinção específica por absorção UV, atividade de água (Aw), teor de clorofilas e carotenoides. Os resultados demonstraram que a infusão permanente de alho desidratado (AOEVD) e do alho in natura (AOEVIN) no azeite de oliva aumentaram a atividade antioxidante das amostras avaliadas, preservaram o teor de compostos fenólicos totais ao longo do armazenamento e retardaram o processo de degradação das clorofilas em relação a amostra controle (AOEVP). A amostra AOEVIN apresentou o maior índice de acidez; o AOEVD apresentou a menor Aw; e o AOEVD e o AOEVIN reduziram o índice de peróxidos em relação ao AOEVP. Assim, a adição de $10 \%$ $(\mathrm{m} / \mathrm{m})$ de alho desidratado ou in natura de forma permanente pode aumentar a capacidade antioxidante, o teor de compostos fenólicos totais e retardar a degradação de clorofilas no azeite de oliva.
\end{abstract}

Palavras-chave: Olea europeae L.; Allium sativum; Antioxidante; Azeite de oliva.

\begin{abstract}
Garlic (Allium sativum) is popularly used to flavor olive oil, it has high levels of allicin and selenium with antioxidant capacity and also phenolic compounds such as p-coumaric and caffeic acids. The present work evaluated the quality of extra virgin olive oil flavored individually with dryed and in natura garlic, both in the proportion of $10 \%$ (w/w) during permanent infusion for 50 days at $60 \pm 2{ }^{\circ} \mathrm{C}$. Analyzes were performed to determine the antioxidant activity, the total phenolic compounds content, the thermal stability and the main quality parameters by determining the acidity index, peroxide index, specific extinction coefficient by UV absorption, water activity (Aw), chlorophylls and carotenoids content. The results showed that the permanent infusion of dehydrated garlic (AOEVD) and fresh garlic (AOEVIN) in olive oil increased the antioxidant activity of the evaluated samples, preserved the content of total phenolic compounds during storage and delayed the degradation process of chlorophylls in relation to the control sample (AOEVP). The AOEVIN sample had the highest acidity index; the AOEVD showed the lowest Aw; the AOEVD and the AOEVIN reduced the peroxide index in relation to AOEVP. The addition of $10 \%$ (w/w) dehydrated or in natura garlic permanently can increase the antioxidant capacity, the phenolic content and delay the degradation of chlorophylls in the olive oil.
\end{abstract}

Keywords: Olea europeae L.; Allium sativum; Antioxidant; Olive oil. 


\begin{abstract}
Resumen
El ajo (Allium sativum) se usa popularmente para aromatizar el aceite de oliva, tiene altos niveles de alicina y selenio con capacidad antioxidante y también compuestos fenólicos como los ácidos p-cumárico y cafeico. El presente trabajo evaluó la calidad del aceite de oliva virgen extra aromatizado individualmente con ajo seco e in natura, ambos en la proporción de $10 \%(\mathrm{~m} / \mathrm{m})$ en infusión permanente durante 50 días a $60 \pm 2^{\circ} \mathrm{C}$. Se realizaron análisis para determinar la actividad antioxidante, el contenido fenólico total, la estabilidad termica y los principales parámetros de calidad mediante la determinación del índice de acidez, índice de peróxidos, coeficiente de extinción específico por absorción UV, actividad del agua (Aw), contenido de clorofilas y carotenoides. Los resultados mostraron que la infusión permanente de ajo deshidratado (AOEVD) y ajo fresco (AOEVIN) en aceite de oliva aumentó la actividad antioxidante de las muestras evaluadas, preservó el contenido de compuestos fenólicos totales durante el almacenamiento y retrasó el proceso de degradación de las clorofilas en relación con la muestra de control (AOEVP). La muestra de AOEVIN tuvo el índice de acidez más alto; el AOEVD tuvo el Aw más bajo; el AOEVD y el AOEVIN redujeron el índice de peróxido en relación al AOEVP. La adición de $10 \%(\mathrm{~m} / \mathrm{m})$ de ajo deshidratado o in natura de forma permanente puede aumentar la capacidad antioxidante, el contenido fenólico y retrasar la degradación de las clorofilas en el aceite de oliva.
\end{abstract}

Palabras clave: Olea europeae L.; Allium sativum; Antioxidante; Aceite de oliva.

\title{
1. Introdução
}

O azeite de oliva representa a principal fonte de lipídios da dieta de países mediterrâneos com impacto na saúde e no bem-estar (Perestrelo et al., 2017). Os benefícios nutricionais devidos ao consumo do azeite de oliva estão relacionados principalmente à sua composição de ácidos graxos, ao alto teor de ácido oleico e à razão equilibrada de ácidos graxos saturados e poli-insaturados (Makni et al., 2015). Os benefícios do azeite na saúde humana são atribuídos principalmente aos compostos fenólicos e a capacidade antioxidante, importantes na prevenção de muitas doenças, como câncer, distúrbios inflamatórios, degeneração neurológica, doenças coronárias e diabetes mellitus tipo 2 (Foscolou, Critselis \& Panagiotakos, 2018; Perestelo et al., 2017; Sacchi et al., 2017). Também são atribuídos aos polifenóis do azeite de oliva as propriedades antialérgicas, antiaterogênicas, antitrombóticas, antimutagênicas, anticâncer, antiangiogênicas, antiapoptóticas e antimicrobianas (GorzynikDebicka et al., 2018; Guo et al., 2018; Ramos et al., 2020).

O azeite de oliva extra virgem é um produto resultante da extração lipídica por processos físicos e mecânicos e possui um alto valor comercial agregado definido por parâmetros de qualidade mínimos e máximos, expressos em legislação específica, como teor de acidez, índice de peróxidos, índice de extinção em ultravioleta e composição de ácidos graxos (Costa, Melloni \& Ferreira, 2019; Brasil, 2018). O uso da espectroscopia UV-Vis (Minuceli et al., 2021) e estudos sobre estabilidade térmica (Cestario et al., 2021) também tem contribuído para análises de adulterações e para garantia da qualidade dos azeites.

Diversas matérias-primas podem ser adicionadas aos azeites de oliva condimentados (Mannina et al., 2012; Yilmazer et al., 2016). A adição destas matérias-primas pode ser feita na forma de óleo essencial, produto seco, in natura ou em diferentes combinações (Baiano et al., 2009, Baiano et al., 2016, Perestelo et al., 2017; Sacchi et al., 2017). Entre os condimentos utilizados, destaca-se o alho (Allium sativum), comumente utilizado para temperar e condimentar alimentos em sua forma in natura ou em combinações com outros ingredientes. O alho possui alto teor de alicina com propriedades antimicrobianas e considerável teor de selênio com ação antioxidante (Felix, Medeiros \& Medeiros, 2018; Fonseca et al., 2014; Shafer \& Kaschula, 2014). Pode ser consumido ou utilizado nas suas diferentes formas de apresentação: in natura, inteiro, triturado, em pasta, em pó, seco ou também como óleo essencial.

Há pouca informação na literatura científica sobre azeites de oliva extravirgem condimentados pela adição de alho em contato direto e permanente com o azeite ao longo da sua vida útil. Assim, essa pesquisa busca avaliar a composição físicoquímica do azeite após a condimentação permanente com alho desidratado e in natura a fim de compreender o papel da aromatização nas mudanças do perfil oxidativo, antioxidante e físico-químico dos azeites de oliva. 


\section{Metodologia}

$\mathrm{O}$ estudo de pesquisa experimental foi desenvolvido para avaliar a qualidade do azeite de oliva extravirgem condimentado por infusão permanente de $10 \%(\mathrm{~m} / \mathrm{m})$ de alho desidratado e $10 \%(\mathrm{~m} / \mathrm{m})$ de alho in natura, conforme forma de preparo e análises oficiais previamente definidas.

\subsection{Matérias-primas}

O azeite de oliva extravirgem de origem portuguesa, das variedades de oliveiras Galega e Cobrançosa, bulbilhos de alho in natura e alho desidratado granulado, da safra de 2020, foram adquiridos no comércio local de Chapecó-SC, Brasil.

\subsection{Preparo do azeite condimentado}

O azeite de oliva condimentado foi preparado pelo método de infusão proposto por Caporaso et al. (2013) com algumas modificações. Foram preparadas amostras de azeite extravirgem condimentado com alho in natura (AOEVIN) a $10 \%$ $(\mathrm{m} / \mathrm{m})$; azeite extravirgem condimentado com alho desidratado (AOEVD) a $10 \%(\mathrm{~m} / \mathrm{m})$ e a amostra controle de azeite extravirgem puro (AOEVP). As amostras foram armazenadas em frascos de vidro âmbar protegidas da exposição à luz. Os condimentos permaneceram em contato com o azeite ao longo de todo o experimento.

\subsection{Análises da oxidação lipídica}

As determinações do índice de acidez (IA), valor de extinção específica no ultravioleta (UV) e determinação do índice de peróxidos (IP) foram realizadas para os azeites de oliva extravirgem com e sem condimentos (AOEVIN, AOEVD, AOEVP), conforme a metodologia dos Anexos II e IX do Regulamento CEE / 2568/1991 da União Europeia (CEE, 1991).

\subsection{Análises da atividade antioxidante e compostos fenólicos totais}

$\mathrm{Na}$ determinação da atividade antioxidante dos azeites foram realizadas as análises da capacidade de sequestro do radical 2,2-Azino-bis (3-etilbenzotiazolina-6-ácido sulfônico) (ABTS) conforme método descrito por Re et al. (1999) e da capacidade de sequestro do radical livre estável 1,1-difenil-2-picrilhidrazil (DPPH·) conforme descrito por Kouka et al. (2017). O conteúdo total de compostos fenólicos totais (CFT) foi determinado de acordo com o procedimento descrito por Singleton, Orthofer \& Lamuela-Raventos (1999).

\subsection{Determinação de clorofila, carotenoides e Aw}

A fração de clorofilas foi medida em espectrofotômetro UV a $670 \mathrm{~nm}$ e a fração de carotenoides a $470 \mathrm{~nm}$, conforme Minguez - Mosquera et al., (1991). A análise de atividade de água (Aw) foi medida em determinador de Aw (Aqualab Pro®) em temperatura ambiente $\left(23-24^{\circ} \mathrm{C}\right)$, conforme orientações do fabricante.

\subsection{Teste de estabilidade térmica}

A estabilidade térmica foi determinada pelo método de Schaal oven test conforme adaptado de Yang et al. (2016) e consistiu em manter por 50 dias a $60 \pm 2^{\circ} \mathrm{C}$ em estufa com circulação constante de ar (CIENLAB, modelo CE - 220/150), as amostras AOEVP, AOEVIN e AOEVD envasadas em frascos âmbar de $60 \mathrm{ml}$. As amostras foram analisadas a cada 10 dias, quanto ao IP, IA, extinção específica no ultravioleta, teor de clorofila, teor de carotenoides e Aw. Também foram avaliadas quanto ao CFT e atividade antioxidante pelos métodos DPPH e ABTS. 


\subsection{Análise estatística}

Todas as determinações analíticas foram realizadas em triplicata. Os valores dos parâmetros foram expressos como a média \pm desvio padrão. As diferenças significativas entre as médias $(\mathrm{p}<0,05)$ foram determinadas pela análise de variância (ANOVA) e a comparação entre as médias pelo Teste de Tukey em nível de 95\% de confiança com uso do software SAS 9.0.

\section{Resultados e Discussão}

\section{1 Índice de acidez e atividade de água}

O IA se manteve estável, sem diferenças significativas ( $p<0,05$ ), para as amostras AOEV e AOEVIN nas análises no tempo zero (0) e até 40 dias de armazenamento e aumentou significativamente $(\mathrm{p}<0,05)$ para a amostra controle AOEVP após 20 dias de armazenamento sob condições do método de Schaal oven test (Tabela 1).

Tabela 1. Índice de acidez titulável para o azeite extravirgem puro e condimentado com diferentes formas de alho avaliado pelo método Schaal oven test a $60 \pm 2^{\circ} \mathrm{C}$ nos tempos $0,10,20,30,40$ e 50 dias.

\begin{tabular}{cccc}
\hline & \multicolumn{3}{c}{ Índice de Acidez (\% ácido oleico) } \\
\hline Tempo (dias) & AOEVP & AOEVD & AOEVIN \\
\hline 0 & $0,30 \pm 0,00^{\mathrm{Dd}}$ & $0,30 \pm 0,00^{\mathrm{Db}}$ & $0,42 \pm 0,02^{\mathrm{Bb}}$ \\
10 & $0,30 \pm 0,00^{\mathrm{Dd}}$ & $0,30 \pm 0,00^{\mathrm{Db}}$ & $0,45 \pm 0,00^{\mathrm{Bb}}$ \\
20 & $0,30 \pm 0,00^{\mathrm{Dd}}$ & $0,30 \pm 0,00^{\mathrm{Db}}$ & $0,45 \pm 0,00^{\mathrm{Bb}}$ \\
30 & $0,32 \pm 0,01^{\mathrm{Cc}}$ & $0,30 \pm 0,00^{\mathrm{Db}}$ & $0,45 \pm 0,05^{\mathrm{Bb}}$ \\
40 & $0,33 \pm 0,01^{\mathrm{Cb}}$ & $0,30 \pm 0,00^{\mathrm{Db}}$ & $0,45 \pm 0,00^{\mathrm{Bb}}$ \\
50 & $0,45 \pm 0,00^{\mathrm{Ba}}$ & $0,45 \pm 0,00^{\mathrm{Ba}}$ & $0,56 \pm 0,02^{\mathrm{Aa}}$ \\
\hline
\end{tabular}

Os valores fornecidos são as médias das três repetições \pm desvio padrão. Resultados com a mesma letra minúscula na mesma coluna não diferem entre si e com a mesma letra maiúscula não diferem entre as amostras em diferentes tempos de análises pelo teste de Tukey (p < 0,05). Azeites de oliva extravirgem: AOEVP = puro (azeite sem condimentação), AOEVD = azeite de oliva extravirgem condimentado com alho desidratado $10 \%$ ( $\mathrm{m} / \mathrm{m}$ ), AOEVIN = azeite de oliva extravirgem condimentado com alho in natura $10 \%(\mathrm{~m} / \mathrm{m})$. Fonte: Autores (2021).

Houve aumento significativo $(\mathrm{p}<0,05)$ do IA para a amostra AOEVIN em relação a amostra AOEVP e AOEVD em todos os tempos avaliados (Tabela 1). Destaca-se que o IA das amostras AOEVP e AOEVD, comparadas entre si, em 50 dias de armazenamento não apresentou diferenças estatísticas e houve aumento significativo do IA para amostra AOEVIN no mesmo tempo (Tabela 1). Todos os resultados obtidos estiveram em conformidade com o limite máximo de acidez de $0,8 \%$ estabelecido pela legislação brasileira e internacional (Brasil, 2018; CEE, 1991). Esses resultados demonstram que a adição de alho in natura aumenta a acidez do azeite extravirgem, provavelmente devido a presença de ácidos orgânicos que podem migrar da matéria-prima adicionada para o azeite e pelo aumento no teor de umidade do azeite condimentado devido a presença de alho fresco, o qual possui cerca de $70 \%$ de umidade, o que pode acelerar reações químicas e enzimáticas que resultam no aumento de ácidos graxos livres (Sousa et al., 2015). Quanto a manutenção da acidez em AOEVD e AOEVIN após 40 dias de armazenamento (Tabela 1), Gambacorta et al. (2007) encontrou resultados parecidos, afirmando que em azeites aromatizados com alho desidratado a acidez mantem-se inalterada por longos períodos de armazenamento.

A Aw inicial foi menor para a amostra AOEVD em relação as amostras AOEVIN e AOEVP e reduziu ao longo do armazenamento para todas as amostras (Figura 1). 
Figura 1. Atividade de água para o azeite extravirgem puro e condimentado com diferentes formas de alho avaliado pelo método Schaal oven test a $60 \pm 2^{\circ} \mathrm{C}$ nos tempos $0,10,20,30,40$ e 50 dias.

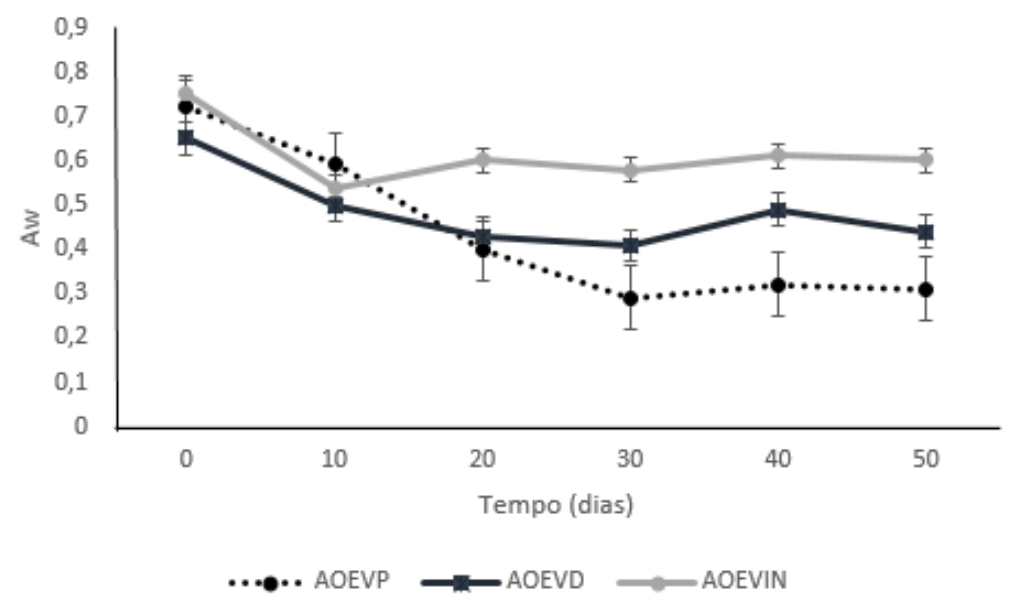

Os valores fornecidos são as médias das três repetições \pm desvio padrão. Azeites de oliva extravirgem: AOEVP = Puro (azeite sem condimentação), AOEVD = azeite de oliva extravirgem condimentado com alho desidratado10 \% (m/m), AOEVIN = azeite de oliva extravirgem condimentado com alho in natura $10 \%$ $(\mathrm{m} / \mathrm{m})$. Fonte: Autores (2021).

Observa-se um aumento na Aw das amostras adicionadas de alho (AOEVD e AOEVIN) em relação ao padrão (AOEVP) após 20 dias de armazenamento (Figura 1). Contudo, todas as amostras apresentaram valores de $0,4<$ Aw $<0,7$. De acordo com a literatura, a Aw ótima para inibir reações químicas e evitar o crescimento microbiológico é de 0,4 e as reações químicas e enzimáticas têm suas velocidades reduzidas com a diminuição da Aw (Welti \& Vergana, 1997). Nesse sentido, a redução da Aw observada ao longo do armazenamento é desejável. A adição de alho desidratado ao azeite de oliva contribuiu para a maior redução da Aw em relação a adição de alho in natura (Figura 1) uma vez que a quantidade de água livre é menor em produtos desidratados o que também contribui para reduzir a oxidação lipídica (Kasimoglu et al., 2018).

\section{2 Índice de peróxidos}

Houve um aumento significativo ( $\mathrm{p}<0,05)$ e crescente para o índice de peróxidos (IP) em todas as amostras analisadas ao longo do período de armazenamento (Figura 2).

Figura 2. Índice de peróxidos para o azeite extravirgem puro e condimentado com diferentes formas de alho pelo método Schaal oven test a $60 \pm 2^{\circ} \mathrm{C}$ nos tempos $0,10,20,30,40$ e 50 dias.

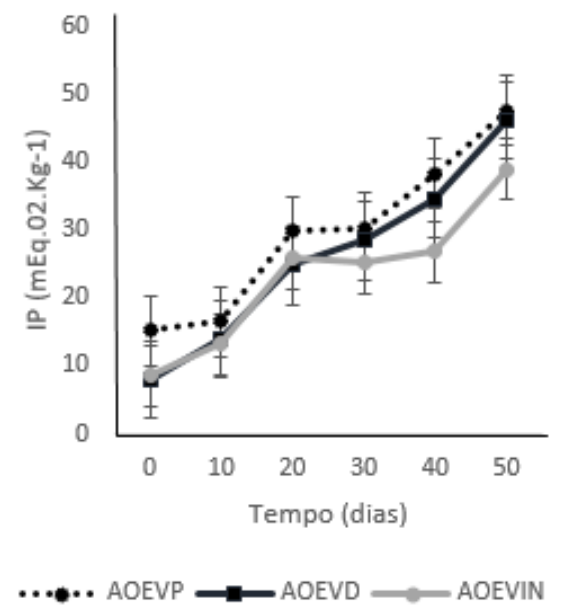

Os valores fornecidos são as médias das três repetições \pm desvio padrão. Azeites de oliva extravirgem: AOEVP = Puro (azeite sem condimentação), AOEVD = azeite de oliva extravirgem condimentado com alho desidratado $10 \%(\mathrm{~m} / \mathrm{m})$, AOEVIN = azeite de oliva extravirgem condimentado com alho in natura $10 \%$ (m/m). Fonte: Autores (2021). 
As amostras AOEVD e AOEVIN apresentaram os menores valores para o IP no tempo zero, em relação ao tratamento AOEVP neste mesmo período (Figura 2). Isso sugere que a adição de alho nas formas in natura e desidratada retarda a formação de compostos de degradação do azeite de oliva extravirgem na fase inicial da condimentação. Embora todas as amostras apresentaram aumento em relação ao IP durante o armazenamento pelo método de Schaal oven test, destaca-se que o AOEVIN apresentou estabilidade e não diferiu estatisticamente $(p<0,05)$ entre 20 e 40 dias de armazenamento (Figura 2). $\mathrm{O}$ AOEVD apresentou estabilidade térmica entre 20 e 30 dias, enquanto o AOEVP apresentou estabilidade térmica entre 10 e 20 dias e teve aumento crescente do IP (Figura 2). Após 20 dias de armazenamento nas condições do método de Schaal oven test, todas as amostras ultrapassaram o limite máximo de IP $\leq 20 \mathrm{mEq} . \mathrm{O} 2 / \mathrm{Kg}$, conforme estabelecido pela legislação brasileira e internacional (CEE, 1991; Brasil, 2018). O IP é o método mais comum para avaliar a estabilidade térmica dos óleos vegetais e o aumento do IP indica a formação de hidroperóxidos durante a oxidação lipídica (Chandran et al., 2017; Keramat et al., 2016). No presente estudo, observa-se que a adição de $10 \%(\mathrm{~m} / \mathrm{m})$ de alho, tanto na forma in natura quanto desidratada, reduziu o IP, o que indica que retardou a oxidação lipídica no azeite de oliva em comparação com a amostra controle (Figura 2).

\subsection{Extinção específica no ultravioleta}

A adição de alho manteve a estabilidade térmica das amostras AOEVD e AOEVIN após 20 e 30 dias de armazenamento sob condições de Schaal oven test em relação ao tratamento controle (AOEVP). Contudo, em todos as amostras observou-se um aumento significativo $(\mathrm{p}<0,05)$ e crescente para os valores de K232 (Figura 3).

Figura 3. Coeficiente de extinção específica em ultravioleta - valores de $K_{232}$ (a) e $K_{270}$ (b) para o azeite extravirgem puro e condimentado com diferentes formas de alho avaliado pelo método Schaal oven test a $60 \pm 2^{\circ} \mathrm{C}$ nos tempos $0,10,20,30,40 \mathrm{e}$ 50 dias.
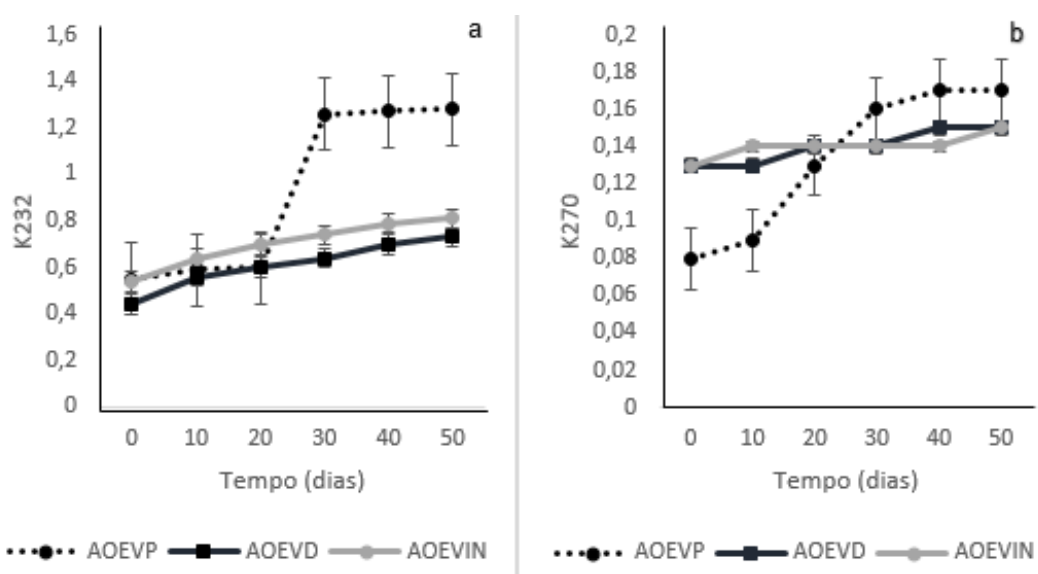

Os valores fornecidos são as médias das três repetições \pm desvio padrão. Azeites de oliva extravirgem: AOEVP $=$ Puro (azeite sem condimentação), AOEVD = azeite de oliva extravirgem condimentado com alho desidratado10 \% (m/m), AOEVIN = azeite de oliva extravirgem condimentado com alho in natura $10 \%$ $(\mathrm{m} / \mathrm{m})$. Fonte: Autores (2021).

O AOEVD apresentou os menores valores de K232 e a maior estabilidade térmica entre 20 e 40 dias de armazenamento (Figura 3). Valores baixos de K232 podem ser encontrados em azeites com alto teor de ácido oleico (C 18:1, ๗-9) ou esteárico (C 18:0), pois estes mostram alta estabilidade oxidativa (Liu, Singh \& Green, 2002). Os valores de K232 e K270, são indicativos da presença de trienos conjugados e de compostos carboxílicos, resultantes de oxidações secundárias. Os valores máximos autorizados para K232 e K270 são, respectivamente, 2,50 e 0,20, conforme o Regulamento 2568/91 (CEE, 1991). Assim, todas as amostras avaliadas estiveram em conformidade com a legislação para os valores de coeficiente de extinção específica em ultravioleta até 50 dias de armazenamento (Figura 3). Mudanças nos valores de K270 são devidas à 
formação de trienos conjugados, bem como compostos insaturados, cetonas e aldeídos (Makni et al., 2015; Sousa et al., 2015). Quando os ácidos linoleico e linolênico são oxidados eles formam hidroperóxidos e as duplas ligações dos óleos se tornam conjugadas e isso aumenta o coeficiente de absorção no ultravioleta (Gray, 1978; AOAC, 2016).

\subsection{Teores de clorofila e carotenoides}

Os resultados expressos na Figura 4 demonstram que os teores iniciais de clorofila são maiores no AOEVP e menores nas amostras AOEVD e AOEVIN. Essa diferença ocorre provavelmente devido ao processo de condimentação.

Figura 4. Teores de clorofila (a) e carotenoides (b) para o azeite extravirgem puro e condimentado com diferentes formas de alho avaliados pelo método Schaal oven test a $60 \pm 2^{\circ} \mathrm{C}$ nos tempos $0,10,20,30,40$ e 50 dias.
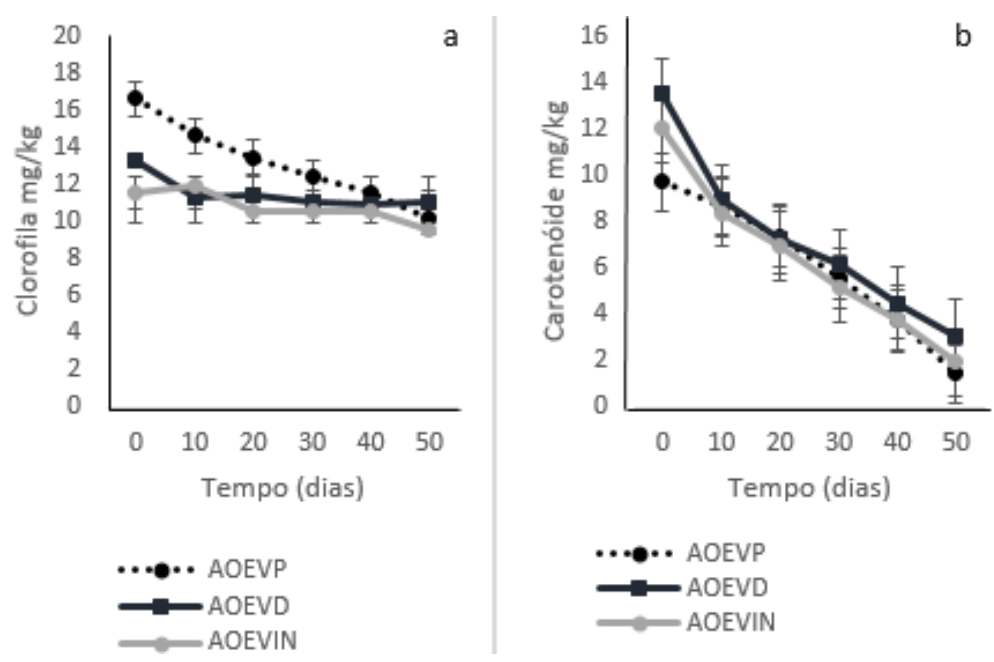

Os valores fornecidos são as médias das três repetições \pm desvio padrão. Azeites de oliva extravirgem: AOEVP $=$ Puro (azeite sem condimentação), AOEVD = azeite de oliva extravirgem condimentado com alho desidratado10 \% (m/m), AOEVIN = azeite de oliva extravirgem condimentado com alho in natura $10 \%$ $(\mathrm{m} / \mathrm{m})$. Fonte: Autores (2021).

A amostra AOEVP apresentou a maior redução do teor de clorofila observada entre o tempo inicial e final de tratamento sob condições de Schaal oven test, em relação as amostras com adição de alho (Figura 4). Contudo, houve maior estabilidade e menor degradação no teor de clorofila para as amostras AOEVD e AOEVIN após 10 dias de armazenamento. Segundo Minguez-Mosquera et al. (1990), quando a atividade enzimática diminui, o teor de pigmento permanece quase constante. As clorofilas podem agir como catalisadores na formação do oxigênio singlete reagindo diretamente com as ligações duplas dos ácidos graxos oleico, linoleico e $\alpha$-linolênico, produzindo espécies reativas de oxigênio (Lanfer-Marquez, Barros, Sinnecker, 2005; Serrano et al., 2016). Contudo, alguns estudos demonstram que as clorofilas podem exercer diversas atividades benéficas, agindo como sequestradoras de radicais livres (Hua Kao, Ju Chen, Huei Chen, 2011; Baiano, Gambacorta, La Notte, 2010).

As amostras condimentadas AOEVD e AOEVIN, apresentaram maiores teores iniciais de carotenoides, porém, todas as amostras apresentaram uma perda no teor de carotenoides superior ao observado no teor de clorofilas (Figura 4). Keramat et al., (2016) relatam o efeito antioxidante do $\beta$-caroteno como um agente protetor contra a oxidação lipídica, porém, destacam que em altas concentrações de oxigênio, o $\beta$-caroteno perde sua atividade e desempenha papel como pró-oxidante.

\subsection{Teor de compostos fenólicos totais e capacidade antioxidante}

Os resultados permitem observar que as amostras AOEVD e AOEVIN apresentaram CFT menor em relação ao AOEVP (Tabela 2). 
Tabela 2. Compostos fenólicos totais (CFT) para o azeite extravirgem puro e condimentado com diferentes formas de alho avaliados pelo método Schaal oven test a $60 \pm 2^{\circ} \mathrm{C}$ nos tempos 0,30 e 50 dias.

\begin{tabular}{|c|c|c|c|}
\hline & & 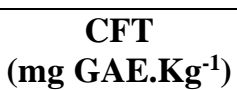 & \\
\hline $\begin{array}{c}\text { Tempo } \\
\text { (dias) }\end{array}$ & AOEVP & AOEVD & AOEVIN \\
\hline $\mathbf{0}$ & $218,55 \pm 0,40^{\mathrm{Aa}}$ & $162,55 \pm 0,70^{\mathrm{Ca}}$ & $173,37 \pm 0,06^{\mathrm{Ba}}$ \\
\hline 30 & $123,76 \pm 0,31^{\mathrm{Fb}}$ & $128,33 \pm 0,21^{\mathrm{Eb}}$ & $134,89 \pm 0,15^{\mathrm{Db}}$ \\
\hline 50 & $111,42 \pm 0,22^{\mathrm{Gc}}$ & $122,85 \pm 0,69^{\mathrm{Fc}}$ & $99,74 \pm 0,68^{\mathrm{Hc}}$ \\
\hline
\end{tabular}

Os valores fornecidos são as médias das três repetições \pm desvio padrão. Resultados com a mesma letra minúscula na mesma coluna não diferem entre si e com a mesma letra maiúscula não diferem entre as amostras em diferentes tempos de análises pelo teste de Tukey (p < 0,05). Azeites de oliva extravirgem: AOEVP = puro (azeite sem condimentação), AOEVD = azeite de oliva extravirgem condimentado com alho desidratado10 \% ( $\mathrm{m} / \mathrm{m})$, AOEVIN = azeite de oliva extravirgem condimentado com alho in natura $10 \%$ (m/m). Fonte: Autores (2021).

Houve redução significativa do conteúdo de CFT para todas as amostras ao longo do armazenamento (Tabela 2). A amostra AOEVIN obteve o maior CFT após 30 dias e a amostra AOEVD após 50 dias de armazenamento quando comparada a amostra AOEVP nas mesmas condições. Este estudo permite observar que a condimentação com $10 \%$ de alho in natura contribuiu para a manutenção do maior teor de CFT em 30 dias de armazenamento e com $10 \%$ de alho desidratado observouse o maior CFT entre as amostras com 50 dias de armazenamento. As diferenças obtidas para o teor de CFT entre as amostras podem ser explicadas com base nas interações que ocorrem entre o azeite e os compostos fenólicos e antioxidantes presentes no alho, como a alicina, selênio, ácidos p-cumárico e cafeico (Aslani et al., 2011; Burian, Sacramento \& Carlos, 2017; Fonseca et al., 2014, Felix, Medeiros \& Medeiros, 2018; Shafer \& Kaschula, 2014) que são capazes de proteger os compostos fenólicos do óleo (Cho \& Xu, 2000; Bozin et al., 2008; Baiano et al., 2009, Caponio et al., 2016).

As amostras AOEVD e AOEVIN apresentaram os maiores valores de atividade antioxidante em relação ao AOEVP e a maior atividade antioxidante foi observada para o tratamento AOEVD após 50 dias de armazenamento (Tabela 3).

Tabela 3. Atividade antioxidante pelos métodos ABTS e DPPH para o azeite extravirgem puro e condimentado com diferentes formas de alho pelo método Schaal oven test a $60 \pm 2^{\circ} \mathrm{C}$ nos tempos 0,30 e 50 dias.

\begin{tabular}{cccc}
\hline \multicolumn{3}{c}{$\left.\left.\begin{array}{c}\text { ABTS } \\
(\mathbf{m m o l} \text { TE. Kg }\end{array}\right)^{-1}\right)$} \\
\hline Tempo (dias) & AOEVP & AOEVD & AOEVIN \\
\hline 0 & $3,34 \pm 0,01^{\mathrm{Hc}}$ & $3,44 \pm 0,04^{\mathrm{Gc}}$ & $3,79 \pm 0,01^{\mathrm{Fc}}$ \\
30 & $5,63 \pm 0,03^{\mathrm{Eb}}$ & $7,57 \pm 0,03^{\mathrm{Bb}}$ & $5,60 \pm 0,01^{\mathrm{Eb}}$ \\
50 & $6,53 \pm 0,02^{\mathrm{Da}}$ & $8,73 \pm 0,01^{\mathrm{Aa}}$ & $6,97 \pm 0,02^{\mathrm{Ca}}$ \\
\hline \multicolumn{4}{c}{ DPPH } \\
\hline Tempo (dias) & $\mathbf{A O E V P}$ & $\mathbf{A O E V D}$ & $\mathbf{A O E V I N}$ \\
\hline 0 & $5,33 \pm 0,01^{\mathrm{Aa}}$ & $5,09 \pm 0,05^{\mathrm{Ba}}$ & $5,09 \pm 0,05^{\mathrm{Ba}}$ \\
30 & $3,27 \pm 0,01^{\mathrm{Fc}}$ & $4,89 \pm 0,01^{\mathrm{Dc}}$ & $4,89 \pm 0,01^{\mathrm{Dc}}$ \\
50 & $4,67 \pm 0,02^{\mathrm{Eb}}$ & $5,01 \pm 0,02^{\mathrm{Cb}}$ & $5,01 \pm 0,02^{\mathrm{Cb}}$ \\
\hline
\end{tabular}

Os valores fornecidos são as médias das três repetições \pm desvio padrão. Resultados com a mesma letra minúscula na mesma coluna não diferem entre si e com a mesma letra maiúscula não diferem entre as amostras em diferentes tempos de análises pelo teste de Tukey (p < 0,05). Azeites de oliva extravirgem: AOEVP = puro (azeite sem condimentação), AOEVD = azeite de oliva extravirgem condimentado com alho desidratado10 \% ( $\mathrm{m} / \mathrm{m}$ ), AOEVIN = azeite de oliva extravirgem condimentado com alho in natura $10 \%(\mathrm{~m} / \mathrm{m})$. Fonte: Autores (2021).

No ensaio de DPPH, houve redução inicial da atividade antioxidante após 30 dias de armazenamento para todas as amostras e a amostra AOEVD obteve os maiores valores para a análise de DPPH em relação as amostras AOEVP e AOEVIN (Tabela 3). As propriedades antioxidantes do alho têm sido atribuídas a componentes organo-sulfurados, formados a partir da decomposição da alicina (Aydin \& Kahyaoglu, 2019). No presente estudo, a adição de alho no azeite aumentou a atividade antioxidante após 30 dias de armazenamento. 


\section{Considerações Finais}

Na presente pesquisa observou-se que a condimentação com a adição de $10 \%(\mathrm{~m} / \mathrm{m})$ de alho nas formas in natura e desidratado reduziu o índice de peróxidos e a formação de compostos de degradação do azeite de oliva extravirgem apenas na fase inicial de armazenamento e não apresentou variação desejável para impedir a peroxidação lipídica em relação a amostra controle não condimentada após 20 dias de armazenamento. Também houve mudança no perfil de extinção específica no ultravioleta para as amostras condimentadas com alho em comparação ao azeite de oliva padrão sem condimentos. Os azeites condimentados com alho in natura e desidratado retardaram o processo de degradação das clorofilas. A condimentação com 10 $\%(\mathrm{~m} / \mathrm{m})$ de alho desidratado e in natura contribuiu para a manutenção do teor de compostos fenólicos totais e aumentou a atividade antioxidante do azeite de oliva ao longo do armazenamento. Trabalhos futuros poderão avaliar o perfil dos principais compostos químicos que migraram do condimento adicionado para o azeite bem como a aceitabilidade sensorial dos azeites condimentados.

\section{Agradecimentos}

Os autores agradecem ao suporte da Fundação de Amparo à Pesquisa do Estado de Santa Catarina - FAPESC 2021 TR854.

\section{Referências}

A.O.A.C. (2016). Association of Official analytical Chemists. Official methods of analysis of AOAC International. $20 \mathrm{ed.}$

Aslani, M. R., Najarnezhad, V., Mohri, M. \& Azad, M. (2011). The effect of allicin on blood and tissue lead content in mice. Comparative Clinical Pathology, $20,121-125$.

Aydin, S. \& Kahyaoglu, D. T. (2020). Antioxidant effect potential of garlic in vitro and real food system: effects of garlic supplementation on oxidation stability and sensory properties of butter. European Journal of Lipid Science and Technology, 122 (3), 1900261-1900273.

Baiano, A., Gambacorta, G. \& La notte, E. (2010). Aromatization of olive oil. Transworld Research Network, 1-29.

Baiano, A., Gambacorta, G., Terracone, C., Previtali, M. A., Lamacchia, C. \& La notte, E. (2009). Changes in phenolic content and antioxidant activity of Italian extra-virgin olive oils during storage. Journal of Food Science, 74 (2), 177-183.

Baiano, A., Previtali, M. A., Viggiani, I., Varva, G., Squeo, G., Paradiso, V. M., Summo, C., Gomes, T. \& Caponio, F. (2016). As oil blending affects physical, chemical, and sensory characteristics of flavoured olive oils. European Food Research and Technology, 242, $1693-1708$.

Bozin, B., Mimica-Dukic, N., Samojlik, I., Goran, A \& Igic, R. (2008). Phenolics as antioxidants in garlic (Allium sativum L., Alliaceae). Food Chemistry, $111,925-929$.

BRASIL. (2018). Ministério da Agricultura, Pecuária e Abastecimento. Instrução Normativa nº 24,18 de junho de 2018 . Regulamento técnico do azeite de oliva e do óleo de bagaço de oliva. Diário Oficial da União, Brasília, DF.

Burian, J. P., Sacramento, L. V. S. \& Carlos, I. Z. (2017). Fungal infection control by garlic extracts (Allium sativum L.) and modulation of peritoneal macrophages activity in murine model of sporotrichosis. Brazilian Journal of Biology, 77 (4), 848-855.

Caponio, F., Durante, V., Varva, G., Silletti, R., Prevital, A., Viggiani, I., Squeo, G., Summo, C., Pasqualone, A., Gomes, T. \& Baiano, A. (2016). Effect of infusion of spices into the oil vs. combined malaxation of olive paste and spices on quality of naturally flavoured virgin olive oils. Food Chemistry, 202, 221228 .

Caporaso, N., Paduano, A., Nicoletti, G. \& Sacchi, R. (2013). Capsaicinoids, antioxidant activity, and volatile compounds in olive oil flavored with dried chili pepper (Capsicum annuum). European Journal Lipid Science Technology, 115, 1434-1442.

CEE (1991). Regulamento da Comissão (ECC) n $n^{0}$ 2568/91: relativo às características do azeite e óleo de bagaço de azeitona e nos métodos de análise pertinentes. Jornal Oficial da União Europeia, L248, 1-82.

Cestario, A. C. de O.; Meira, K. U.; Contiero, R. L.; Rosa, C. I. L. F. (2021). Quality of olive oils and olive bagasse oil and their use in thermal processes. Research, Society and Development, 10 (2), 1-8.

Chandran, J., Nayana, N., Roshini, N. \& Nisha, P. (2017). Oxidative stability, thermal stability and acceptability of coconut oil flavored with essential oils from black pepper and ginger. Journal of Food Science and Technology, 54, 144-152.

Cho, B.H.S. \& Xu, S. (2000). Effects of allyl mercaptan and various allium-derived compounds on cholesterol synthesis and secretion in Hep-G2 cells, Comparative Biochemistry and Physiology Part C: Pharmacology, Toxicology and Endocrinology, 126 (2), $195-201$. 
Costa, S. M. L., Melloni, R. \& Ferreira, G. M. R. (2019). Potencial biotecnológico de microrganismos do solo na olivicultura do brasil: uma revisão. Revista em Agronegócio e Meio Ambiente, 12 (2), 723.

Felix, A. L. M., Medeiros, I. L. \& Medeiros, F. D. (2018). Allium Sativum: uma nova abordagem frente a resistência microbiana - uma revisão. Brazilian Journal of Health, 1 (2), 201-207.

Fonseca, G. M., Passos, T. C., Ninahuaman, M. F.M.L., Caroci, A. S. \& Costa, L. S. (2014). Avaliação da atividade antimicrobiana do alho (Allium sativum Liliaceae) e de seu extrato aquoso. Revista Brasileira de Plantas Medicinais, 16, 679-684.

Foscolou, A., Critselis, E. \& Panagiotakos, D. (2018). Olive oil consumption and human health: A narrative review. Maturitas, 118, 60-66.

Gambacorta, G., Faccia, M., Pati, S., Lamacchia, C., Baiano, A. \& La Notte, E. (2007). Changes in the chemical and sensorial profile of extra virgin olive oils flavored with herbs and spices during storage. Journal of Food Lipids, 14, 202-215.

Gorzynik-Debicka, M., Przychodzen, P., Capello, F., Kuban-Jankowska, A., Gammazza, A. M., Knap, N., Wozniak, M. \& Gorska-Ponikowska, M. (2018). Potential health benefits of olive oil and plant polyphenols. International Journal of Molecular Sciences, $19,1-13$.

Gray, J. I. (1978). Measurement of lipid oxidation: a review. Journal of the American Oil Chemist's Society, 55, 538-546.

Guo, Z., Jia, X., Zheng, Z., Lu, X., Zheng, Y., Zheng, B. \& Xiao, J. (2018). Chemical composition and nutritional function of olive (Olea europea L.): a review. Phytochemistry Reviews, 17, 1091-1110.

Hua Kao, T., Ju Chen, C. \& Huei Chen, B. (2011). An improved high-performance liquid chromatography - photodiode array detection - atmospheric pressure chemical ionization - mass spectrometry method for determination of chlorophylls and their derivatives in freeze-dried and hot-air-dried Rhinacanthus nasutus (L.) Kurz. Talanta, 86, 349-355.

Kasimoglu, Z., Tontul, I., Soylu, A., Gulen, K. \& Topuz, A. (2018). The oxidative stability of flavoured virgin olive oil: the effect of the water activity of Rosemary. Journal of Food Measurement and Characterization, 12, 2080-2086.

Keramat, M., Golmakani, M., Aminlari, M. \& Shekarforoush, S. S. (2016). Comparative effect of Bunium persicum and Rosmarinus officinalis essential oils and their synergy with citric acid on the oxidation of virgin olive oil. International Journal of Food Properties, 19 (12), $2666-2681$.

Kouka, P., Priftis, A., Stagos, D., Angelis, A., Stathopoulos, P., Xynos, N., Skaltsoumis, A., Mamoulakis, C., Tsatsakis, A. \& Spandidos, D. A. (2017). Assessment of the antioxidant activity of an olive oil total polyphenolic fraction and hydroxytyrosol from a Greek Olea europea variety in endothelial cells and myoblasts. International Journal of Molecular Medicine, 40, 708-712.

Lanfer-Marquez, U. M., Barros, R. M. C. \& Sinnecker, P. (2005). Antioxidant activity of chlorophylls and their derivatives. Food Research International, 38, $885-891$.

Liu, Q., Singh, S. \& Green, A. (2002). High-oleic and high-stearic cottonseed oils: nutritionally improved cooking oils developed using gene silencing. Journal of American College of Nutrition, 21, 205-211.

Makni, M., Haddar, A., Fraj, A. B. \& Zeghal, N. (2015). Physico-chemical properties, composition, and oxidative stability of olive and soybean oils under different conditions. International Journal of Food Properties, 18, 194-204.

Mannina, L., D'imperio, M., Gobbino, M., D'amico, I., Casini, A., Emanuele, M. C. \& Sobolev, A. P. (2012). Nuclear magnetic resonance study of flavoured olive oils. Flavour and Fragrance Journal, 27, 250-259.

Minguez-Mosquera, M. I. (1991). Determination of chlorophylls and carotenoids by high performance liquid chromatography during olive lactic fermentation. Journal of Chromatography, 585, 259-266.

Minuceli, F. da S.; Silva, J. M. da; Silveira, R. da; Santos, O. O. (2021). UV-VIS methodology for the quantification of vegetable oil in adulterated olive oil. Research, Society and Development, 10(6), 1-8.

Perestrelo, R., Silva, C., Silva, P. \& Câmara, J. S. (2017). Global volatile profile of virgin olive oils flavoured by aromatic/medicinal plants. Food Chemistry, $227,111-121$.

Ramos, A. C.; Negreiros, J. H. C. N.; Lima, A. K. M. de; Cordeiro, M. de A.; Godoy, G. P. (2020). The therapeutic applicability of tyrosol and hydroxytyrosol for Dentistry. Research, Society and Development, 9 (8), 1-16.

Re, R., Pellegrini, N., Proteggente, A., Pannala, A., Yang, M. \& Rice-Evans, C. (1999). Antioxidant activity applying an improved ABTS radical cation decolorization assay. Free Radical, Biology \& Medicine, 26, 1231-1237.

Sacchi, R., Medaglia, D. D., Paduano, A., Caporaso, N. \& Genovese, A. (2017). Characterisation of lemon-flavoured olive oils. LWT - Food Science and Technology, 79, 326-332.

Serrano, L., Cruz, A., Sousa, S. \& Morais, Z. (2016). Alterations in monovarietal, blended and aromatized Portuguese virgin olive oils under four storage conditions for 12 months. European Food Research and Technology, 242 (7), 1041-1055.

Shäfer, G. \& Kaschula, C. H. (2014). The immunomodulation and anti-inflammatory effects of garlic organosulfur compounds in cancer chemoprevention. Anti-Cancer Agents in Medicinal Chemistry, 14 (2), 233- 240.

Singleton, V. L., Orthofer, R. \& Lamuela-Raventos, R. M. (1999). Analysis of total phenols and other oxidation substrates and antioxidants by means of FolinCiocalteau reagent. Methods in Enzimology, 299, 152-178.

Sousa, A., Casal, S., Malheiro, R., Lamas, H., Bento, A. \& Pereira, J. A. (2015). Aromatized olive oils: Influence of flavouring in quality, composition, stability, antioxidants, and antiradical potential. LWT - Food Science and Technology, 60 (1), 22-28. 
Research, Society and Development, v. 10, n. 15, e412101522945, 2021

(CC BY 4.0) | ISSN 2525-3409 | DOI: http://dx.doi.org/10.33448/rsd-v10i15.22945

Welti, J. \& Vergana, F. (1997). Atividade de água/ Conceito y aplicación em alimentos com alto contenido de humedad. In: Aguilera, J.M. Temas em Tecnologia de alimentos. Santiago, Chile, 1, 11-26.

Yang, Y., Song, X., Sui, X., Qi, B., Wang, Z., Li, Y. \& Jiang, L. (2016). Rosemary extract can be used as a synthetic antioxidant to improve vegetable oil oxidative stability. Industrial Crops and Products, 80, 141-147.

Yilmazer, M., Goksu, S., Ozkan, G. \& Karacabey, E. (2016). Aroma transition from rosemary leaves during aromatization of olive oil. Journal of Food and Drug Analysis, 24, 299-304. 